\title{
QUALIDADE DE VIDA NO TRABALHO DE PESSOAS COM DEFICIÊNCIA FÍSICA
}

\author{
QUALITY OF LIFE AT WORK OF PEOPLE WITH DISABILITIES
}

\section{CALIDAD DE VIDA EN EL TRABAJO DE PERSONAS CON DEFICIENCIA FÍSICA}

\author{
Bertran Gonçalves Coutinho ${ }^{1}$ \\ Inacia Sátiro Xavier de França ${ }^{2}$ \\ Alexsandro Silva Coura ${ }^{3}$ \\ Kaio Keomma Aires Silva Medeiros ${ }^{4}$ \\ Jamilly da Silva Aragão ${ }^{5}$
}

Resumo Objetivou-se avaliar a qualidade de vida no trabalho e os principais fatores correlacionados em pessoas com deficiências físicas. Tratou-se de estudo transversal, realizado em 2012, em serviço de referência em reabilitação de pessoas com deficiência, localizado em João Pessoa, na Paraíba. A amostra foi composta por 110 indivíduos que responderam a um questionário sociodemográfico; utilizou-se uma escala validada para avaliar a qualidade de vida no trabalho. Para análise dos dados, efetuaram-se os testes Alfa de Cronbach, Kaiser-Meyer-Olkin e esfericidade de Bartlett. O índice de consistência interna da escala se mostrou satisfatório $(\alpha=0,85)$, atestando a confiabilidade do instrumento utilizado. A matriz de correlações entre os itens da escala revelou a possibilidade de sua fatorialização (KMO =0,60) e Bartlett $(\mathrm{p}<0,001)$. Quanto à percepção da qualidade de vida no trabalho, $67,9 \%$ indicaram insatisfação; $21,4 \%$, avaliação intermediária; e 10,7\%, satisfação. Os fatores mais correlacionados foram: salário $(0,74)$, capacidade de ascensão profissional $(0,73)$, oportunidade de expressar suas opiniões $(0,71)$, carga horária e quantidade de trabalho $(0,66)$. Concluiu-se que a qualidade de vida no trabalho das pessoas com deficiência física não é satisfatória, principalmente em razão de aspectos como salário, carga horária e quantidade de trabalho inadequados, bem como dificuldade para ascensão profissional.

Palavras-chave pessoas com deficiência; satisfação no trabalho; condições de trabalho; local de trabalho.
Abstract This study aimed to evaluate the quality of life at work and the main correlated factors for people with disabilities. This was a cross-sectional study carried out in 2012 at a reference center for the rehabilitation of people with disabilities located in the city of João Pessoa, the state capital of Paraíba, Brazil. The sample consisted of 110 individuals who answered a sociodemographic questionnaire; a validated scale was used to assess the quality of life at work. Cronbach's Alfa test, the Kaiser-Meyer-Olkin test, and Bartlett's sphericity test were done for data analysis. The level of internal consistency of the scale was satisfactory $(\alpha=0.85)$, attesting to the reliability of the instrument that was used. The correlation matrix between scale items showed the possibility for factor analysis $(\mathrm{KMO}=0.60)$ and Bartlett $(\mathrm{p}<0.001)$. Insofar as views on the quality of life at work is concerned, $67.9 \%$ pointed to dissatisfaction; $21.4 \%$, had an intermediate evaluation, and $10.7 \%$ claimed to be satisfied. The most correlated factors were salary $(0.74)$, possibility for professional growth $(0.73)$, opportunity to express opinions (0.71), hour load and amount of work (0.66). It was concluded that the quality of life at work of people with disabilities is not satisfactory, mainly due to issues such as salary, working hours, inadequate amounts of work, and hurdles in career advancement. Keywords people with disabilities; job satisfaction; work conditions; workplace. 


\section{Introdução}

O trabalho, cada vez mais, torna-se importante na vida das pessoas, exigindo-se maior dedicação para as atividades laborais. No entanto, em alguns casos, o ato de trabalhar não é prazeroso, porque se manifesta de forma fragmentada e com diversas exigências, tornando-se conflitante com a vida social ou familiar (Descanio e Lunardelli, 2007). Nesse contexto, os modelos modernos de tecnologias de gestão enfatizam a qualidade de vida no trabalho (QVT) e os aspectos relacionados com a satisfação do indivíduo em sua atividade laboral, tendo-se registro do uso público desta expressão nos anos 1970, nos Estados Unidos, assumindo, àquela época, uma definição ampla e empírica, com reflexos ainda nos dias atuais - uma vez que há mais de uma conceituação disponível na literatura acerca desse construto (Figueiredo et al., 2009).

Apesar das diferentes conceituações ora apresentadas, pode-se compreender a qualidade de vida no trabalho como "a aplicação concreta de uma filosofia que busca alterar aspectos do trabalho, a fim de criar uma situação mais favorável à satisfação das necessidades dos trabalhadores e ao aumento da produtividade organizacional" (Kurogi, 2008, p. 54). De outro ponto de vista, pode-se defini-la como “a capacidade de administrar um conjunto de ações, incluindo diagnóstico, implantação de melhorias e inovações gerenciais, tecnológicas e estruturais no trabalho, com prioridade para o bem-estar das pessoas da organização" (Limongi-França, 2007, p. 167).

Mais objetivamente, pode-se dizer que a QVT é a humanização do trabalho, pois envolve o atendimento às necessidades do trabalhador, o aumento do seu bem-estar e a reestruturação das formas de organizar o trabalho com maior participação dos trabalhadores (Kirogi, 2008). Engloba, pois, aspectos físicos, psicossociais e legislativos, que devem ser alcançados a fim de proporcionar bem-estar físico, mental, econômico e social, permitindo que o indivíduo usufrua a sua condição de cidadão - e sinta-se, portanto, respeitado e valorizado no ambiente de trabalho (Freitas e Souza, 2009).

Assim, quadro se inserem as pessoas com deficiências (PsDs), particularmente após a lei n. 8.213, que data de 1991 e estabelece a obrigatoriedade de as empresas contratarem e incluírem esses indivíduos, observando proporções específicas e considerando o número total de funcionários - até 20, 2\%; de 201 a 500, 3\%; de 501 a 1.000, 4\%; de 1.001 ou mais, 5\% (Brasil, 1991). Crê-se, no entanto, que não raras vezes os candidatos a uma vaga não conseguem a contratação, ora porque as suas desvantagens e limitações são incompatíveis com o exercício da função oferecida, ora porque não possuem a capacitação necessária para o cargo, devido à carência de instituições de ensino adequadas aos moldes da educação inclusiva.

Com isso, algumas instituições se dedicam a reabilitar, capacitar e encaminhar as PsDs para contratação pelas empresas, identificando as potencia- 
lidades laborativas e implementando um processo orientado que lhes permita o desenvolvimento profissional suficiente para desempenhar suas funções ou outras diferentes das que exerciam no mercado de trabalho (Brasil, 1999). Entretanto, desconhece-se uma prática contínua de acompanhamento dessas pessoas contratadas para avaliação de suas condições no exercício laboral. Nesse sentido, o estudo aqui apresentado buscou resposta para a questão: como as PsDs físicas contratadas por empresas pelo sistema de cotas percebem a sua qualidade de vida no trabalho?

Os indicadores para avaliar a satisfação do ser humano inserido em um sistema produtivo são instrumentos de planejamento e decisão que trazem objetividade de avaliação, facilitam o levantamento de informações e demonstram dados e efeitos de determinadas ações. Para se estudarem os indicadores de QVT, foram propostos modelos, ao longo dos anos, que levaram os nomes de seus autores: Walton (1973), Hackman e Oldhan (1975) e Nadler e Lawler (1983). O primeiro é um dos mais utilizados no Brasil, pela sua clareza, objetividade e, ainda, por priorizar questões elementares à realização do trabalho, enfatizando condições físicas, aspectos relacionados à segurança, fatores higiênicos, bem como remuneração (Timossi et al., 2009).

O estudo aqui apresentado partiu do pressuposto de que dificuldades enfrentadas por PsDs no mercado de trabalho, como lacunas no desenvolvimento de programas de emprego, critérios inadequados na elaboração de avaliações laborais e espaço físico e logístico excludentes no ambiente laboral (Oliveira, Goulart Júnior e Fernandes, 2009), podem prejudicar a QVT; razão pela qual objetiva-se avaliar a qualidade de vida dessas pessoas no trabalho e os principais fatores correlacionados.

O estudo também procurou contribuir, com seus resultados, para o preenchimento de lacunas no que concerne à identificação das condições de trabalho e de qualidade de vida enfrentadas pelas PsDs. Ademais, os resultados poderão subsidiar o planejamento de ações educativas, visando ampliar o conhecimento das empresas contratantes sobre segurança, saúde e legislação no trabalho, na perspectiva de minimizar as dificuldades enfrentadas por esses trabalhadores.

\section{Métodos}

O estudo foi do tipo transversal, com abordagem quantitativa, desenvolvido em 2012 na Fundação Centro Integrado de Apoio ao Portador de Deficiência (Funad), serviço de referência em reabilitação de PsDs na cidade de João Pessoa, Paraíba.

A instituição é constituída por equipe interdisciplinar e está dividida em coordenadorias, entre as quais umas delas é a Coordenadoria de Treinamento, Produção e Ensino Profissionalizante (Corpu), local onde os dados foram coletados e que dispõe de um arquivo onde ficam armazenadas as informações 
dos usuários avaliados, as quais são encaminhadas às empresas que solicitam assessoria da fundação.

A amostra foi calculada pela fórmula: $\mathrm{n}=\mathrm{N}$. Z2 . P (1 - P) / (N-1) . e2 + $\mathrm{Z2}$. ( 1 - P), em que: $\mathrm{n}=$ valor da amostra; $\mathrm{N}=$ valor da população; $\mathrm{Z}=$ intervalo de confiança $(1,96) ; \mathrm{P}=$ prevalência; $\mathrm{e}=$ erro tolerado $(0,05)$, obtendo-se um n probabilístico de 110 sujeitos, com correção de $5 \%$ para compensar eventuais perdas ou problemas operacionais da pesquisa. Participaram da pesquisa indivíduos com idade igual ou superior a 18 anos, independentemente de sexo, nível econômico, social e tipo de deficiência física, avaliados pelo setor de triagem da Funad/Corpu, beneficiados com a Lei de Cotas (lei n. 8.213/1991), residentes e com emprego formal na cidade de João Pessoa, além de sua cognição preservada.

Para a coleta de dados, aplicaram-se um questionário sociodemográfico e uma escala validada para avaliar QVT, sendo esta última do tipo Likert, polarizada em cinco pontos: 1 (muito insatisfeito), 2 (insatisfeito), 3 (nem insatisfeito nem satisfeito), 4 (satisfeito), 5 (muito satisfeito). A proposta dela é avaliar a satisfação do colaborador em relação ao critério indicado sobre a QVT, levando em conta suas necessidades e anseios (Timossi et al., 2009).

Os dados foram analisados com auxílio do software Statistical Package for Social Science (SPSS) 18.0, utilizando-se dos testes: análise fatorial exploratória de componentes principais (para exame da relação entre as variáveis); Kaiser-Meyer-Olkin com parâmetro de adequação >0,5 (avaliação da proporção da variância dos dados, que pode ser considerada comum a todas as variáveis); teste de esfericidade de Bartlett (para verificar a adequação dos dados para a realização da análise fatorial); e, finalmente, o teste Alfa de Cronbach com limiar >0,70 (verificação de consistência interna da escala). Ademais, no que se refere, especificamente, à pontuação dos participantes na escala de QVT, consideraram-se os critérios sugeridos pelos autores de validação do instrumento: $x \leq 2-$ Insatisfatório, $2>x>4$-Intermediário, $\mathrm{x} \geq 4$-Satisfatório (Timossi et al., 2009).

Todos os participantes assinaram o Termo de Consentimento Livre e Esclarecido e foram informados da possibilidade de desistirem da pesquisa em qualquer fase, sem penalização. Esta pesquisa foi analisada e aprovada pelo Comitê de Ética em Pesquisa da Universidade Estadual da Paraíba (CAAE n. 0194.0.133.000-11).

\section{Resultados}

\section{Perfil sociodemográfico}

Conforme a Tabela 1, em que são apresentados os dados sociodemográfi$\cos$ dos participantes, verificou-se que na maior parte eram homens $(69,1 \%)$, 
adultos jovens $(43,6 \%)$, tendo até o ensino médio completo $(77,3 \%)$ e com baixa renda $(54,5 \%)$.

\begin{tabular}{|c|c|c|}
\hline \multicolumn{3}{|c|}{$\begin{array}{l}\text { Perfil sociodemográfico de pessoas com deficiência física - Funad, } \\
\text { João Pessoa (PB), } 2012\end{array}$} \\
\hline Variáveis & $\mathrm{N}$ & $\%$ \\
\hline \multicolumn{3}{|l|}{ Sexo } \\
\hline Masculino & 76 & 69,1 \\
\hline Feminino & 34 & 30,9 \\
\hline \multicolumn{3}{|l|}{ Faixa etária } \\
\hline $18-27$ anos & 48 & 43,6 \\
\hline $28-37$ anos & 44 & 40 \\
\hline $38-47$ anos & 17 & 15,5 \\
\hline $48-57$ anos & 01 & 0,9 \\
\hline \multicolumn{3}{|l|}{ Escolaridade } \\
\hline Fundamental incompleto & 05 & 4,5 \\
\hline Fundamental completo & 17 & 15,5 \\
\hline Médio incompleto & 19 & 17,3 \\
\hline Médio completo & 44 & 40 \\
\hline Superior incompleto & 09 & 8,2 \\
\hline Superior completo & 16 & 14,5 \\
\hline \multicolumn{3}{|l|}{ Renda familiar } \\
\hline Sem renda & 16 & 14,5 \\
\hline 1-2 salários mínimos & 44 & 40 \\
\hline 2-3 salários mínimos & 26 & 23,7 \\
\hline 3-4 salários mínimos & 11 & 10 \\
\hline > 5 salários mínimos & 13 & 11,8 \\
\hline
\end{tabular}

Fonte: Os autores.

\section{Teste de consistência da escala de QVT}

Na Tabela 2, verificou-se a correlação total de itens da escala de QVT corrigidos $>0,30$ e Alfa de Cronbach com item deletado $>0,70$. Conforme se pode observar, com Alfa de Cronbach total de 0,85 , atestou-se a consistência interna do instrumento para a amostra estudada. 
Tabela 2

Escala da qualidade de vida no trabalho (QVT) testada com o Alfa de Cronbach - Funad, João Pessoa (PB), 2012

\begin{tabular}{lccc}
\hline \multicolumn{1}{c}{ Itens da escala QVT } & $\begin{array}{c}\text { Correlação total de } \\
\text { itens corrigidos }\end{array}$ & $\begin{array}{c}\text { Alfa de Cronbach } \\
\text { com item deletado }\end{array}$ & $\begin{array}{c}\text { Alfa de Cronbach } \\
\text { total }\end{array}$ \\
\hline Salário & 0,65 & 0,84 & 0,85 \\
Alimentação & 0,30 & 0,85 & 0,85 \\
Vale-transporte & 0,35 & 0,84 \\
Auxílio-saúde & 0,52 & 0,84 \\
Carga horária & 0,58 & 0,84 \\
Quantidade de trabalho & 0,60 & 0,85 \\
Uso de equipamentos que facilitem o trabalho & 0,39 & 0,84 \\
Ambiente de trabalho: acessibilidade/segurança & 0,49 & 0,84 \\
Oportunidade de tomar decisões & 0,55 & 0,85 \\
Ter conhecimento do próprio desempenho & 0,36 & 0,84 \\
Cursos de capacitação e treinamento & 0,47 & 0,83 \\
Capacidade de ascensão profissional & 0,65 & 0,85 \\
Estabilidade trabalhista & 0,32 & 0,84 \\
Discriminação & 0,42 & 0,85 \\
Relacionamento com colegas e superiores & 0,34 & 0,85 \\
Recebe atenção especial quando solicitada & 0,37 & 0,84 \\
Respeito aos direitos do trabalhador & 0,51 & 0,84 \\
Oportunidade de expressar suas opiniões & 0,64 & 0,85 \\
Privacidade & 0,34 & 0,84 \\
Tratamento da empresa com os funcionários & 0,52 & \\
\hline
\end{tabular}

Fonte: Os autores.

\section{Análise fatorial dos componentes da QVT}

No tocante à QVT, verificou-se que 21,4\% $(\mathrm{n}=12)$ dos participantes relataram insatisfação; 67,8\% (n=38), satisfação intermediária; e o menor contingente, 10,7\% (n=6), satisfação. A Tabela 3 aponta a correlação entre os escores obtidos em relação à satisfação dos entrevistados e os componentes da escala da QVT. 
Tabela 3

\begin{tabular}{|c|c|}
\hline Itens da escala QVT & Fator \\
\hline Salário & 0,74 \\
\hline Capacidade de ascensão profissional & 0,73 \\
\hline Oportunidade de expressar suas opiniões & 0,71 \\
\hline Carga horária & 0,66 \\
\hline Quantidade de trabalho & 0,66 \\
\hline Oportunidade de tomar decisões & 0,63 \\
\hline Respeito aos direitos do trabalhador & 0,62 \\
\hline Tratamento da empresa com os funcionários & 0,60 \\
\hline Auxílio-saúde & 0,56 \\
\hline Ambiente de trabalho: acessibilidade/segurança & 0,56 \\
\hline Cursos de capacitação e treinamento & 0,54 \\
\hline Discriminação & 0,50 \\
\hline Recebe atenção especial quando solicitada & 0,48 \\
\hline Estabilidade trabalhista & 0,45 \\
\hline Ter conhecimento do próprio desempenho & 0,43 \\
\hline Vale-transporte & 0,40 \\
\hline Alimentação & 0,36 \\
\hline Relacionamento com colegas e superiores & 0,28 \\
\hline Privacidade & 0,21 \\
\hline Uso de equipamentos que facilitem o trabalho & 0,18 \\
\hline
\end{tabular}

Fonte: Os autores.

Nota: itens ordenados de acordo com a magnitude de suas cargas fatoriais:

Eigenvalue $=5,90 ; \%$ de variância explicada $=29,54$.

\section{Discussão}

A análise da demografia das PsDs partícipes do presente estudo revelou algumas características relevantes, a começar pelo maior quantitativo de indivíduos do sexo masculino. Como os participantes dessa investigação atenderam ao critério de elegibilidade que previa a inserção no mercado de trabalho, tal observação também apontou maior número de homens com deficiência empregados, se comparado com o quantitativo de mulheres divergindo, pois, do que se tem observado em algumas pesquisas realizadas no Brasil (Vasconcelos, 2010; Baptista e Silva, 2010). 
Apesar de os dados relativos à inserção de PsDs no mercado formal de trabalho serem recentes, pode-se dizer que esses sujeitos ocupam menos de $1 \%$ dos empregos formais no Brasil, considerando a Relação Anual de Informações Sociais (Rais), apontando uma realidade ainda longe da ideal, sobretudo para mulheres, uma vez que estas ocupam aproximadamente a metade das vagas se comparadas com aquelas preenchidas por homens (Brasil, 2014).

Acredita-se, pois, que mulheres com deficiência vivenciam um duplo preconceito: pela deficiência que, invariavelmente, lhes impõe limitações de ordem física e também social; bem como pela carga estigmatizante, tendo em vista a histórica exclusão da mulher no mercado de trabalho. Crê-se, portanto, que esses fatores influenciam e, por vezes, potencializam a pouca oferta de vagas de emprego para essas mulheres.

Apesar de os dados referentes à distribuição de empregos entre PsDs do sexo masculino e do feminino divergirem de alguns estudos, outros dados sociodemográficos identificados na pesquisa aqui apresentada se coadunam com a literatura, pois se verificou que a maioria entre aqueles que executavam alguma atividade laboral era de adultos jovens, com ensino médio completo e baixa renda (Pereira e Passerino, 2012; Vasconcelos, 2010; Baptista e Silva, 2010).

A despeito da renda, especificamente, sabe-se que no Brasil, do total das PsDs, 29,05\% vivem em situação de miséria, com menos de um salário mínimo por mês (Santos, 2011), o que estabelece um importante vínculo entre a deficiência e a pobreza, conforme aponta estudo sobre políticas públicas no mercado de trabalho para esse grupo populacional, não apenas no referido país, mas também nos Estados Unidos e em nações da União Europeia (Oliveira, Goulart Júnior e Fernandes, 2009).

No que se refere à satisfação pelo exercício de uma dada atividade laboral, acredita-se que ela pode estar relacionada com um conjunto de sentimentos favoráveis ou desfavoráveis com os quais os trabalhadores percebem seu trabalho, sujeitando-se à influência de forças internas e externas, o que pode afetar a saúde do trabalhador. Ainda assim, alguns aspectos sempre são fundamentais para se alcançar um nível mínimo de satisfação no trabalho, e um deles é o aspecto financeiro.

Destarte, a variável que mais influenciou a QVT dos entrevistados foi o salário. É possível considerar, com isso, que a remuneração pode ser um fator gerador de insatisfação profissional. Embora não deva haver qualquer diferenciação, sabe-se que PsDs acabam recebendo valores menores em relação às pessoas sem deficiência, sendo que as desvantagens encontradas pelos primeiros acabam por condená-los a condições de trabalho desfavoráveis e rendimentos particularmente baixos (França, Pagliuca e Baptista, 2008; Souza e Carneiro, 2007; Lino e Cunha, 2008). 
Apesar de o salário figurar como o componente mais relacionado com uma satisfatória QVT de PsDs, adicionalmente observou-se, pelos resultados do estudo, que outros fatores são, igualmente, muito importantes nesse contexto, tais como: capacidade de ascensão profissional; carga horária e quantidade de trabalho; e oportunidade de expressar suas opiniões.

Com relação à ascensão profissional, constatou-se que, no Brasil, a maioria de trabalhadores com deficiência desempenhava funções operacionais, raramente ocupando um cargo mais elevado, o que pode ser explicado pela falta de investimento na capacitação funcional da organização e pela descrença dos empregadores quanto à capacidade das PsDs de assumirem cargos de liderança. Acerca dessa questão, uma pesquisa mostrou que em 27 países eles, quando em idade produtiva, experimentavam desvantagens significativas e piores resultados no mercado de trabalho do que pessoas sem deficiência (World Health Organization, 2011).

O referido estudo constatou que as PsDs estão em desvantagem no mercado de trabalho provavelmente devido à falta de acesso à educação e ao treinamento, recursos de financiamento, natureza do ambiente de trabalho e percepção de seus empregadores acerca da deficiência (Organização Mundial da Saúde, 2012). Por tudo, crê-se que a qualificação/capacitação profissional daquelas pessoas certamente é um dos caminhos a serem enfrentados para que possam ascender na função (Leonardi et al., 2012; Pereira, Del Prette e Del Prette, 2009).

No tocante à carga horária e à quantidade de trabalho, aspectos que, assim como a ascensão profissional, influenciaram sobremaneira a QVT para os participantes do estudo aqui apresentado, sabe-se que, em geral, um regime de quarenta horas semanais favorece o cumprimento de um grande número de atividades, mas, adicionalmente, demanda muita dedicação. Além disso, solicita um esforço físico que pode prejudicar a saúde das pessoas com algum tipo de deficiência, aspectos que podem ser observados em algumas pesquisas relacionadas ao tema, as quais apontam que a quantidade e a sobrecarga de trabalho resultam em fatores causadores de insatisfação profissional, devendo ser considerados em políticas na área (Figueiredo et al., 2009; Siqueira e Kurcgant, 2012). No entanto, essas questões podem, em alguns casos, levar os empregadores a ver PsDs como menos produtivas e mais onerosas (Souza e Carneiro, 2007).

No que concerne ao último componente mais intimamente relacionado à QVT, oportunidade de expressar suas opiniões, pode-se inferir que as PsDs acabam sendo discriminadas pelos empregadores, assim como pelos colegas, por causa de conceitos errôneos sobre suas capacidades, reforçando uma visão preconceituosa acerca delas. 


\section{Conclusão}

A identificação de que homens com algum tipo de deficiência ocupam mais vagas no mercado de trabalho do que as mulheres, apesar de estas últimas serem maioria no contingente populacional de PsDs, o que foi verificado no estudo aqui apresentado e é corroborado pela literatura, sugere que estudos sejam conduzidos para analisar os fatores determinantes dessa realidade.

Ademais, a QVT das PsDs não é satisfatória, principalmente em razão dos fatores de salário, carga horária e quantidade de trabalho inadequados e dificuldade para ascensão profissional, os quais precisam ser levados em consideração no planejamento de políticas na área trabalhista, para os setores públicos e privados, a fim de que tal realidade seja superada.

Crê-se, por fim, que seja necessário maior incentivo à implementação de uma cultura de fomento às pesquisas que objetivem compreender a inserção das PsDs no mercado formal de trabalho, uma vez que o tema é atual e ainda pouco explorado pela comunidade acadêmica.

\section{Colaboradores}

Todos os autores contribuíram na concepção e no delineamento da pesquisa; na análise e interpretação dos dados; na redação do artigo e sua revisão crítica; e na aprovação da versão a ser publicada. Não há conflitos de interesse. 
Resumen Se apuntó a evaluar la calidad de vida en el trabajo y los principales factores correlacionados, en personas con deficiencias físicas. Se trató de un estudio transversal, realizado en 2012, en un servicio de referencia en rehabilitación de personas con deficiencia, ubicado en João Pessoa, capital del estado de Paraíba, Brasil. La muestra se componía de 110 individuos que respondieron a un cuestionario sociodemográfico; se utilizó una escala validad para evaluar la calidad de vida en el trabajo. Para el análisis de los datos, se realizaron las pruebas Alfa de Cronbach, Kaiser-Meyer-Olkin y esfericidad de Bartlett. El índice de consistencia interna de la escala se mostró satisfactorio $(\alpha=0,85)$, acreditando la confiabilidad del instrumento utilizado. La matriz de correlaciones entre los elementos de la escala reveló la posibilidad de su factoración ( $\mathrm{KMO}=0,60)$ y Bartlett $(\mathrm{p}<0,001)$. En cuanto a la percepción de la calidad de vida en el trabajo, el 67,9\% señaló insatisfacción; el 21,4\%, evaluación intermedia; y el 10,7\%, satisfacción. Los factores más correlacionados fueron: salario $(0,74)$, capacidad de ascenso profesional $(0,73)$, oportunidad de expresar sus opiniones $(0,71)$, carga horaria y cantidad de trabajo $(0,66)$. Se concluyó que la calidad de vida en el trabajo de las personas con deficiencia física no es satisfactoria, principalmente en razón de aspectos como salario, carga horaria y cantidad de trabajo inadecuados, así como dificultad de ascenso profesional.

Palabras clave personas con deficiencia; satisfacción en el trabajo; condiciones de trabajo; local de trabajo.

\section{Notas}

1 Faculdade Maurício de Nassau, Campina Grande, Paraíba, Brasil.

<bertranrad@yahoo.com.br>

Correspondência: Rua Francisco Calixto, Monte Santo, CEP 58400-750, Campina Grande, Paraíba, Brasil.

2 Universidade Estadual da Paraíba, Programa de Pós-Graduação em Enfermagem, Departamento de Enfermagem, Campina Grande, Paraíba, Brasil.

<isxf@oi.com.br>

3 Universidade Estadual da Paraíba, Programa de Pós-Graduação em Saúde Pública, Departamento de Enfermagem, Campina Grande, Paraíba, Brasil.

$<$ alex@uepb.edu.br>

4 Universidade de São Paulo, Faculdade de Saúde Pública, São Paulo, São Paulo, Brasil. $<$ keomma.kaio@gmail.com>

5 Universidade Estadual da Paraíba, Programa de Pós-Graduação em Saúde Pública, Campina Grande, Paraíba, Brasil.

<jamilly_aragao@hotmail.com> 


\section{Referências}

BAPTISTA, Ana K.; SILVA, Fabiana C. M. Perfil demográfico e do emprego das pessoas com deficiência no município de Belo Horizonte, Minas Gerais - Brasil. Revista Médica de Minas Gerais, Belo Horizonte, v. 20, n. 1, p. 37-48, 2010.

BRASIL. Lei n. 8.213, de 24 de julho de 1991. Dispõe sobre os planos de benefícios da previdência social e dá outras providências. Diário Oficial da União, Brasília, DF, 25 jul. 1991. Disponível em: <www.planalto. gov.br/ccivil_03/leis/L8213cons.htm>. Acesso em: 12 de Jan de 2017.

BRASIL. Decreto n. 3.298, de 20 de dezembro de 1999. Regulamenta a lei n. 7.853, de 24 de outubro de 1989, dispõe sobre a Política Nacional para a Integração da Pessoa Portadora de Deficiência, consolida as normas de proteção, e dá outras providências. Diário Oficial da União, Brasília, DF, 21 dez. 1999. Disponível em: <www.planalto. gov.br/ccivil_03/decreto/d3298.htm>. Acesso em: 12 jan. 2017.

BRASIL. Ministério do Trabalho e Emprego. Relação social de informação social. Brasília: Ministério do Trabalho e Emprego, 2014. Disponível em: < http://portalfat.mte.gov.br/ wp-content/uploads/2016/03/Característicasdo-Emprego-Formal-segundo-a-RelaçãoAnual-de-Informações-Sociais-201431082014.pdf>. Acesso em: 12 jan. 2017

DESCANIO, Denise; LUNARDELLI, Maria C. Saúde e qualidade de vida na empresa familiar. Psicologia: Organizações e Trabalho, Brasília, v. 7, n. 1, p. 159-177, 2007.

FIGUEIREDO, Isadora M. et al. Qualidade de vida no trabalho: percepções dos agentes comunitários de equipes de saúde da família. Revista Enfermagem Uerj, Rio de Janeiro, v. 17, n. 2, p. 262-267, 2009.

FRANÇA, Inacia S. X.; PAGLIUCA, Lorita M. F.; BAPTISTA, Rosilene S. Política de inclusão do portador de deficiência: possibi- lidades e limites. Acta Paulista de Enfermagem, São Paulo, v. 21, n. 1, p. 112-116, 2008.

FREITAS, André L. P.; SOUZA, Rennata G. B. Um modelo para avaliação da qualidade de vida no trabalho em universidades públicas. Sistemas \& Gestão, Niterói, v. 4, n. 2, p. 136-154, 2009.

HACKMAN, Richard; OLDHAM, Greg R. Development of the Job Diagnostic Survey. Journal of Applied Psychology, Washington, v. 60, n. 2, p. 159-170, 1975.

KUROGI, Marcia S. Qualidade de vida no trabalho e suas diversas abordagens. Revista de Ciências Gerenciais, Londrina, v. 12, n. 16, p. 49-62, 2008.

LEONARDI, Matilde et al. A population survey in Italy based on the ICF classification: recognizing persons with severe disability. The Scientific World Journal, Nova York, v. 2012, 9 p., 2012. Disponível em <www. hindawi.com/journals/tswj/2012/189097>. Acesso em: 12 de Jan de 2017

LIMONGI-FRANÇA, Ana C. Práticas de recursos humanos-PRH: conceitos, ferramentas e procedimentos. São Paulo: Atlas, 2007.

LINO, Michelle V.; CUNHA, Ana C. B. Uma questão de cotas? Como pessoas com deficiência percebem sua inserção no mercado de trabalho com base em políticas públicas de inclusão. Pesquisas e Práticas Psicossociais, São João Del-Rei, v. 3, n. 1, p. 65-74, 2008.

NADLER, David A.; LAWLER, Eward E. Quality of work life: perspectives and directions. Organizational Dynamics, Nova York, v. 11, n. 3, p. 20-30, 1983.

OLIVEIRA, Marileide A.; GOULART JÚNIOR, Edward; FERNANDES, José M. Pessoas com deficiência no mercado de trabalho: considerações sobre políticas públicas nos Estados Unidos, União Europeia e Brasil. Revista Bra- 
sileira de Educação Especial, Marília, v. 15, n. 2, p. 219-232, 2009.

WORLD HEALTH ORGANIZATION (WHO). World Report on disability. Genebra: WHO/The World Bank, 2011. Disponível em: <www. who.int/disabilities/world_report/2011/ report.pdf $>$. Acesso em: 12 jan. 2017.

PEREIRA, Ana C. C.; PASSERINO, Liliana. Um estudo sobre o perfil dos empregados com deficiência em uma organização. Revisa Brasileira de Educação Especial, Marília, v. 18, n. 2, p. 245-264, 2012.

PEREIRA, Camila S.; DEL PRETTE, Almir; DEL PRETTE, Zilda A. P. Habilidades sociais de trabalhadores com e sem deficiência física. Psicologia: Teoria e Pesquisa, Brasília, v. 25, n. 3, p. 339-346, 2009

SANTOS, Wederson R. Deficiência e BPC: o que muda na vida das pessoas atendidas? Ciência \& Saúde Coletiva, Rio de Janeiro, v. 16, n. 1, p. 787-796, 2011.

SIQUEIRA, Vera T. A.; KURCGANT, Paulina. Satisfação no trabalho: indicador de qualidade no gerenciamento de recursos humanos em enfermagem. Revista da Escola de Enfermagem da USP, São Paulo, v. 46, n. 1, p. 151-157, 2012.
SOUZA, José M.; CARNEIRO, Ricardo. Universalismo e focalização na política de atenção à pessoa com deficiência. Saúde e Sociedade, São Paulo, v. 16, n. 3, p. 69-84, 2007.

TIMOSSI, Luciana S. et al. Adaptação do modelo de Walton para avaliação da qualidade de vida no trabalho. Revista da Educação Física/UEM, Maringá, v. 20, n. 3, p. 395-405, 2009.

VASCONCELOS, Fernando D. O trabalhador com deficiência e as práticas de inclusão no mercado de trabalho de Salvador, Bahia. Revista Brasileira de Saúde Ocupacional, São Paulo, v. 35, n. 121, p. 41-52, 2010.

WALTON, Richard E. Quality of Working Life: What is it? Sloan Management Review, Cambridge, v. 15, n. 1, p. 11-21, 1973.

Recebido em 23/04/2015

Aprovado em 04/07/2016 